\title{
A GENERALIZATION OF K. T. CHEN'S INVARIANTS FOR PATHS UNDER TRANSFORMATION GROUPS \\ BY
}

H. H. JOHNSON(1)

In a series of papers $[1 ; 2 ; 3 ; 4], \mathrm{K}$. T. Chen introduced and studied certain infinite series of numbers associated with paths in Euclidean $n$-space. These numbers were invariants under translations, and in [4] he proved that they uniquely characterize paths under translations.

This paper's purpose is investigating similar path invariants for other transformation groups. Integral invariants are studied using prolongations in the context of C. Ehresmann's "jets" [5].

A general discussion of the ideas involved is given in $\$ 1$. $\$ 2$ is devoted to differential equations and some illuminating examples. We find in $\$ 3$ a sufficient condition that a group's invariants uniquely characterize paths. The theory also applies to pseudo groups having sufficiently regular behavior. All structures assumed $C^{\infty}$ (infinitely differentiable).

\section{INVARIANTS}

1.1. Prolongations. Let $M$ be a real $C^{\infty}$-manifold. Let $G$ be a Lie group acting effectively as a transformation group of $C^{\infty}$-homeomorphisms on $M$. If $g \in G$, then $g: M \rightarrow M$. Let $\Theta=\{\theta\}$ be the Lie algebra of vector fields on $M$ induced by the Lie algebra of $G$.

$R$ is the real line, $t \in R, x \in M$. A $k$-jet $j_{t}^{k}(\tau)$ with source $t=\alpha\left(j_{t}^{k}(\tau)\right)$ and target $x=\beta\left(j_{t}^{k}(\tau)\right)$ is the equivalence class of all $C^{\infty}$-functions $\tau$ mapping a neighborhood of $t$ into $M$, which satisfy $\tau(t)=x$ and have the first same $k$ derivatives at $t$. If $\left(x^{1}, \cdots, x^{n}\right)$ are local coordinates on a neighborhood $U$ of $x$, this $k$-jet will be specified by numbers $t, x^{i}, x_{1}^{i}, x_{2}^{i}, \cdots, x_{k}^{i} ; i=1, \cdots, n$, where

$$
x_{h}^{i}=\frac{d^{h} x^{i}(\tau(t))}{d t^{h}}
$$

$J^{k}(M)$ will denote the $k$-jets of $M$. Then $\left(t, x^{1}, \cdots, x^{n}, x_{1}^{1}, \cdots, x_{1}^{n}, \cdots, x_{k}^{1}, \cdots, x_{k}^{n}\right)$ are local coordinates for $J^{k}(M)$ on $\beta^{-1}(U)$. There are natural $C^{\infty}$-projections $\rho_{k}^{k+r}: J^{k+r}(M) \rightarrow J^{k}(M)$, namely, $\rho_{k}^{k+r}\left(j_{t}^{k+r}(\tau)\right)=j_{t}^{k}(\tau)$.

If $g: M \rightarrow M$, there is a natural prolongation $p^{k}(g): J^{k}(M) \rightarrow J^{k}(M)$, namely, $p^{k}(g)\left(j_{t}^{k}(\tau)\right)=j_{t}^{k}(g \circ \tau)$. Hence $G$ can be prolonged to a transformation group on

Received by the editors July 13, 1961.

(1) Supported by OOR Contract DA-36-ORD-2164. 
$J^{k}(M)$. Vector fields $\theta=\left(d g_{t} / d t\right)_{t=0}$ on $M$ are prolonged to $p^{k}(\theta)=\left(d p^{k}\left(g_{t}\right) / d t\right)_{t=0}$ on $J^{k}(M)$. Let $p^{k}(\Theta)=\left\{p^{k}(\theta) \mid \theta \in \Theta\right\}$.

Similarly, if $h: R \rightarrow R$, there is a prolongation $p^{k}(h)\left(j_{t}^{k}(\tau)\right)=j_{h(t)}^{k}\left(\tau \circ h^{-1}\right)$. This corresponds to changing the variable $t$. Vector fields $\phi$ on $R$ may thus be prolonged to vector fields $p^{k}(\phi)$ on $J^{k}(M)$.

Later, exact expressions in local coordinates will be found for these prolongations. If $g: M \rightarrow M$ and $h: R \rightarrow R$, then $p^{k}(g) \circ p^{k}(h)=p^{k}(h) \circ p^{k}(g)$. Hence if $\theta$ and $\phi$ are vector fields on $M$ and $R$ respectively, then $\left[p^{k}(\theta), p^{k}(\phi)\right]=0$. ([ ] denotes the Lie bracket.)

1.2. Paths. A path in $M$ is a $C^{\infty}-$ map $\tau:[a, b] \rightarrow M$. If $\lambda:[c, d] \rightarrow[a, b]$ is $C^{\infty}$ and increasing, $\tau \circ \lambda$ is considered equivalent to $\tau$. There is a natural prolongation of $\tau$ to $p^{k}(\tau):[a, b] \rightarrow J^{k}(M)$ defined by $p^{k}(\tau)(t)=j_{t}^{k}(\tau)$.

If $t$ denotes the coordinate on $R, \alpha^{*}(d t)$ on $J^{k}(M)$ will be denoted more shortly by $d t$.

Definition 1.1. A 1 -form on an open set of $J^{k}(M)$ of the type Adt is invariant under $G$ when for every $\theta \in \Theta$ or vector field $\phi$ on $R$, the Lie derivative of $A d t$ with respect to $p^{k}(\theta)$ and $p^{k}(\phi)$ is zero. Note that if $\psi$ is a vector field on $J^{k}(M)$, the Lie derivative of $A d t$ with respect to $\psi$ is

$$
[\psi, A d t]=\langle\psi, d A\rangle d t+A d(\langle\psi, d t\rangle) .
$$

This implies that $p^{k}(g) *(A d t)=A d t$ for all $g \in G$.

1.3. Chen constants. The Chen constants for a path $\tau:[a, b] \rightarrow M$ with respect to 1 -forms $A_{1} d t, A_{2} d t, \cdots, A_{r} d t$ on $J^{k}(M)$ are defined to be the infinite set of numbers

$$
\int_{\tau} A_{i_{1}} d t \cdots A_{i_{j}} d t, \quad 1 \leqq i_{j} \leqq r ; j=1,2, \cdots
$$

These integrals are defined inductively by

$$
\int_{\tau} A_{i} d t=\int_{a}^{b}\left(p^{k}(\tau)\right) *\left(A_{i} d t\right)=\int_{a}^{b} A_{i}\left(j_{t}^{k}(\tau)\right) d t
$$

and if $\tau_{t}=\tau \mid[a, t]$,

$$
\int_{\tau} A_{i_{1}} d t \cdots A_{i_{j}} d t=\int_{a}^{b}\left[\int_{\tau_{t}} A_{i_{1}} d t \cdots A_{i_{j-1}} d t\right]\left(p^{k}(\tau)\right) *\left(A_{i_{j}} d t\right)
$$

Following Chen [4], these $A_{1} d t, \cdots, A_{r} d t$ define an "exponential" mapping $\theta$ on paths to the space of formal, noncommutative power series in indeterminants $X_{1}, \cdots, X_{r}$ over $R$ :

$$
\theta(\tau)=1+\sum_{j=1}^{\infty} \Sigma\left(\int_{\tau} A_{i_{1}} d t \cdots A_{i_{j}} d t\right) X_{i_{1}} \cdots X_{i_{j}} .
$$


(These integrals are of course defined only when $p^{k}(\tau)([a, b])$ is contained in the domains of $A_{1} d t, \cdots, A_{r} d t$.)

THEOREM 1.1. Let $A_{1} d t, \cdots, A_{r} d t$ be invariant under $G$. If $\tau$ is any path in $M$, $g$ any element of $G$, then the paths $\tau$ and $g \circ \tau$ have the same Chen constants.

Proof.

$$
\begin{aligned}
\int_{g \circ \tau} A_{j} d t & =\int_{a}^{b} A_{j}\left(j_{t}^{k}(g \circ \tau)\right) d t=\int_{a}^{b} A_{j} \circ p^{k}(g)\left(j^{k}(\tau)\right) d t \\
& =\int_{a}^{b} A_{j}\left(j_{t}^{k}(\tau)\right) d t=\int_{\tau} A_{j} d t
\end{aligned}
$$

Since $(g \circ \tau)_{t}=g \circ \tau_{t}$, equality for the other Chen constants also follows.

\section{Differential EQuations}

Next we study the partial differential equations satisfied by invariant 1 -forms $A d t$. All functions and forms are still assumed to be infinitely differentiable.

2.1. Formal derivatives. The following construction is due to $M$. Kuranishi. A 1-form $\omega$ on an open subset of $J^{k}(M)$ is canonical when $\left(p^{k}(\tau)\right) *(\omega)=0$ for all paths $\tau$. Let $\Omega^{k}$ denote the set of canonical 1 -forms. Take local coordinates $\left(x^{1}, \cdots, x^{n}\right)$ on the open set $U$ in $M$. Then on $\beta^{-1}(U), J^{k}(M)$ has local coordinates $\left(t, x^{i}, x_{1}^{i}, \cdots, x_{k}^{i}\right), i=1, \cdots, n$. If $\tau:(t) \rightarrow\left(x^{i}(t)\right)$, then $p^{k}(\tau):(t) \rightarrow\left(t, x^{i}(t), d x^{i}(t) / d t\right.$, $\left.\cdots, d^{k} x^{i}(t) / d t^{k}\right)$.

Consider the 1 -forms $\pi_{h}^{i}=d x_{h}^{i}-x_{h+1}^{i} d t ; h=1, \cdots, k-1$, and $\pi_{0}^{i}=d x^{i}-x_{1}^{i} d t$; $i=1, \cdots, n$, on $\beta^{-1}(U)$.

$$
\left(p^{k}(\tau)\right) *\left(\pi_{h}^{i}\right)=d\left(\frac{d^{h} x^{i}}{d t^{h}}\right)-\frac{d^{h+1} x^{i}}{d t^{h+1}} d t=0 .
$$

Hence $\pi_{h}^{i} \in \Omega^{k}$ for $h=0,1, \cdots, k-1$. In fact, it may be shown that any $\left.\omega \in \Omega^{k}\right|_{U}$ is $\equiv 0$ modulo $\left\{\pi_{h}^{i}\right\}$ over the real-valued functions.

If $g \in G$ and $\omega \in \Omega^{k}$, and $\tau$ is any path in $M$, then $\left(p^{k}(\tau)\right) *\left(\left(p^{k}(g)\right) *(\omega)\right)=\left(p^{k}(g \circ \tau)\right)$ $*(\omega)=0$, since $g \circ \tau$ is a path in $M$. Hence, $\Omega^{k}$ is invariant.

It follows that if $\theta \in \Theta$ is defined on an open subset of $U$, then $\left[p^{k}(\theta), \pi_{h}^{i}\right] \equiv 0$ modulo $\left\{\pi_{h}^{i}\right\}$. Similar reasoning shows that if $\phi$ is a vector field on $R$, then $\left[p^{k}(\phi), \pi_{h^{\prime}}^{i^{\prime}}\right] \equiv 0$ modulo $\left\{\pi_{h^{\prime}}^{i^{\prime}}\right\}$.

If $F$ is a real-valued $C^{\infty}$-function on an open subset $V$ of $J^{k}(M)$, then on $\left(\rho_{k}^{k+1}\right)^{-1}(V)$ we define $\partial F$ by

$$
d F \equiv(\partial F) d t \text { modulo }\left(\Omega^{k+1}\right) .
$$

When $V \subset \beta^{-1}(U)$,

$$
\partial F=\frac{\partial F}{\partial t}+\frac{\partial F}{\partial x^{j}} x_{1}^{j}+\ldots+\frac{\partial F}{\partial x_{k}^{j}} x_{k+1}^{j} .
$$


The following lemmas may be proved by induction using the canonical forms $\left\{\pi_{h}^{i}\right\}$.

LEMMA 2.1. If $\theta=\theta^{j}\left(\partial / \partial x^{j}\right)$ is a vector field on $U$, then

$$
p^{k}(\theta)=\theta+\sum_{h=1}^{k} \partial^{h} \theta^{j} \frac{\partial}{\partial x_{h}^{j}}
$$

LEMMA 2.2. If $\phi=\xi(t)(\partial / \partial t)$ is a vector field on $R$, then on $\beta^{-1}(U)$,

$$
p^{k}(\phi)=\phi-\sum_{h=1}^{k} \frac{d^{h} \xi}{d t^{h}}\left[\sum_{m=h}^{k}\left(\begin{array}{l}
m \\
h
\end{array}\right) x_{m-h+1}^{j} \frac{\partial}{\partial x_{m}^{j}}\right] \text {. }
$$

2.2. Differential equations for integral invariants. Let $A d t$ be an invariant 1 -form on an open subset of $\beta^{-1}(U)$. If the Lie algebra $\Theta$ is generated by $\theta_{\lambda}=\theta_{\lambda}^{j}\left(\partial / \partial x^{j}\right), \lambda=1, \cdots, \lambda_{0}, A$ must satisfy

$$
0=\left[p^{k}\left(\theta_{\lambda}\right), A d t\right]=\left\langle p^{k}\left(\theta_{\lambda}\right), d A\right\rangle d t+A d\left(\left\langle p^{k}\left(\theta_{\lambda}\right), d t\right\rangle\right),
$$

or,

$$
\theta_{\lambda}^{j} \frac{\partial A}{\partial x^{j}}+\sum_{h=1}^{k} \partial^{h} \theta_{\lambda}^{j} \frac{\partial A}{\partial x_{h}^{j}}=0, \quad \lambda=1, \cdots, \lambda_{0} .
$$

Similarly, for every choice of $\xi(t)$ on $R, A$ must satisfy

$$
0=\xi \frac{\partial A}{\partial t}-\sum_{h=1}^{k} \frac{d^{h} \xi}{d t^{h}}\left[\sum_{m=h}^{k}\left(\begin{array}{c}
m \\
h
\end{array}\right) x_{m-h+1}^{j} \frac{\partial A}{\partial x_{m}^{j}}\right]+A \frac{d \xi}{d t} .
$$

Taking successively $\xi=1, t, t^{2}, \cdots$, one obtains

$$
\begin{aligned}
\frac{\partial A}{\partial t} & =0 \\
A & =\sum_{m=1}^{k} m x_{m}^{j} \frac{\partial A}{\partial x_{m}^{j}} \\
\sum_{m=h}^{k}\left(\begin{array}{l}
m \\
h
\end{array}\right) x_{m-h+1}^{j} \frac{\partial A}{\partial x_{m}^{j}} & =0, \quad h=2, \cdots, k .
\end{aligned}
$$

Thus, the following theorem is true.

THEOREM 2.1. The 1-form Adt defined on an open subset of the coordinate neighborhood $\beta^{-1}(U)$ is invariant under $G$ if and only if equations $\left(\mathrm{E}_{1}\right)$ and and $\left(\mathrm{E}_{2}\right)$ hold.

It is natural to ask whether more information could be gained by studying more general invariant 1 -forms on $J^{k}(M)$. The following may be proved by induction on $k$. 
Proposition 2.1. If

$$
\omega=A d t+A_{j} d x^{j}+\sum_{h=1}^{k} A_{j}^{h} d x_{h}^{j}
$$

is invariant on $\beta^{-1}(U)$ in $J^{k}(M)$, then

$$
\omega^{\prime}=\left(A+A_{j} x_{1}^{j}+\sum_{h=1}^{k} A_{j}^{h} x_{h+1}^{j}\right) d t
$$

is invariant on $J^{k+1}(M)$.

2.3. Complete integrability. Instead of solving equations $\left(E_{1}\right)$ and $\left(E_{2}\right)$ it is customary $[6, \S 75]$ to introduce a new variable $V$ and a new system $\left(E^{\prime}\right)$ with $V$ as an unknown function of $A, t, x^{i}, x_{1}^{i}, \cdots, x_{k}^{i} ; i=1, \cdots, n$; namely,

$\left(E^{\prime}\right)$

$$
\begin{aligned}
\theta_{\lambda}^{j} \frac{\partial V}{\partial x^{j}}+\sum_{h=1}^{k} \partial^{h} \theta_{\lambda}^{j} \frac{\partial V}{\partial x_{h}^{j}} & =0, \quad \lambda=1, \cdots, \lambda_{0}, \\
\frac{\partial V}{\partial t} & =0
\end{aligned}
$$

$$
\begin{aligned}
& A \frac{\partial V}{\partial A}+\sum_{h=1}^{k} h x_{h}^{j} \frac{\partial V}{\partial x_{h}^{j}}=0, \\
& \sum_{m=h}^{k}\left(\begin{array}{c}
m \\
h
\end{array}\right) x_{m-h+1}^{j} \frac{\partial V}{\partial x_{m}^{j}}=0, \quad h=2, \cdots, k .
\end{aligned}
$$

THEOREM 2.2. The system $\left(\mathrm{E}^{\prime}\right)$ is completely integrable.

Proof. The following lemmas may be proved by induction arguments, after which the theorem can be proved by direct computation.

Let $F$ be a real-valued $C^{\infty}$-function on an open subset of $U$.

LEMMA 2.3. If $h \leqq k$,

$$
h \partial^{h} F=\sum_{m=1}^{k} m x_{m}^{i} \frac{\partial\left(\partial^{h} F\right)}{\partial x_{m}^{i}} .
$$

LEMMA 2.4.

$$
\partial^{h} F=\frac{\partial F}{\partial x_{h}^{j}} x_{h}^{j}+G
$$

where $G$ is a function on $J^{h-1}(M)$.

Lemma 2.5 .

$$
\sum_{q=m}^{k}\left(\begin{array}{l}
q \\
m
\end{array}\right) x_{q-m+1}^{j} \frac{\partial\left(\partial^{h} F\right)}{\partial x_{q}^{j}}=\left\{\begin{array}{l}
0 \text { if } h<m, \\
\left(\begin{array}{l}
h \\
m
\end{array}\right) \partial^{h-m+1} F \text { if } h \geqq m .
\end{array}\right.
$$


2.4. Examples. (a) Translations. Here, $M=E^{n}=$ Euclidean $n$-space. $\Theta$ is generated by $\left\{\partial / \partial x^{i} \mid i=1, \cdots, n\right\} . p^{k}(\Theta)=\Theta$. When $k=1,\left(\mathrm{E}^{\prime}\right)$ becomes

$$
\begin{aligned}
\frac{\partial V}{\partial t} & =\frac{\partial V}{\partial x^{i}}=0 ; \quad i=1, \cdots, n, \\
A \frac{\partial V}{\partial A}+x_{1}^{j} \frac{\partial V}{\partial x_{1}^{j}} & =0,
\end{aligned}
$$

having solutions $V^{j}=x_{1}^{j} / A ; j=1, \cdots, n$. Hence the invariant 1 -forms on $J^{1}(M)$ are $x_{1}^{j} d t$. The Chen constants of paths with respect to these invariant 1 -forms are the original invariants found by Chen.

(b) Motions. Let $M=E^{2}=\left\{\left(x^{1}, x^{2}\right)\right\}$. $\Theta$ is generated by $\partial / \partial x^{1}, \partial / \partial x^{2}$, and $x^{2}\left(\partial / \partial x^{1}\right)-x^{1}\left(\partial / \partial x^{2}\right) . p^{2}(\Theta)$ is generated by $\partial / \partial x^{1}, \partial / \partial x^{2}$, and $x^{2}\left(\partial / \partial x^{1}\right)$ $-x^{1}\left(\partial / \partial x^{2}\right)+x_{1}^{2}\left(\partial / \partial x_{1}^{1}\right)-x_{1}^{1}\left(\partial / \partial x_{1}^{2}\right)+x_{2}^{2}\left(\partial / \partial x_{2}^{1}\right)-x_{2}^{1}\left(\partial / \partial x_{2}^{2}\right)$. For $k=2$ equations $\left(\mathrm{E}^{\prime}\right)$ yield two independent solutions,

$$
\frac{\left(\left(x_{1}^{1}\right)^{2}+\left(x_{1}^{2}\right)^{2}\right)^{1 / 2}}{A}, \quad \frac{x_{1}^{1} x_{2}^{2}-x_{1}^{2} x_{2}^{1}}{A\left[\left(x_{1}^{1}\right)^{2}+\left(x_{1}^{2}\right)^{2}\right]} .
$$

Hence if $d s$ denotes arc length and $1 / \rho$ denotes curvature, the invariant 1 -forms on $J^{2}(M)$ are generated by $\left(\left(x_{1}^{1}\right)^{2}+\left(x_{1}^{2}\right)^{2} d t\right)^{1 / 2}=d s$ and

$$
\left\{\left(x_{1}^{1} x_{2}^{2}-x_{1}^{2} x_{2}^{1}\right) /\left[\left(x_{1}^{1}\right)^{2}+\left(x_{1}^{2}\right)^{2}\right]\right\} d t=1 / \rho d s .
$$

In [4] Chen proved that the invariant 1-forms of example (a) are complete, i.e., they essentially characterize paths under translations. This cannot be true in general, especially if one allows the paths to have isolated discontinuities, as Chen does. For example,

$$
\tau_{1}(t)=\left\{\begin{array}{ll}
(t, 0), & 0 \leqq t \leqq 1 / 2, \\
(1-t, 0), & 1 / 2 \leqq t \leqq 1
\end{array} \quad \text { and } \tau_{2}(t)=\{(t, 0) \mid 0 \leqq t \leqq 1\}\right.
$$

have the same Chen constants with respect to $d s$ and $(1 / \rho) d s$, but cannot be obtained from one another by motions. To show that this completeness may fail no matter how large $k$ may be taken, consider the next example.

(c) Special linear group. $M=E^{2}, \Theta$ is generated by

$$
\frac{\partial}{\partial x^{1}}, \quad \frac{\partial}{\partial x^{2}}, x^{2} \frac{\partial}{\partial x^{1}}, x^{1} \frac{\partial}{\partial x^{2}}, x^{1} \frac{\partial}{\partial x^{1}}-x^{2} \frac{\partial}{\partial x^{2}} .
$$

For any $k$, equations $\left(\mathrm{E}_{1}\right)$ and $\left(\mathrm{E}_{2}\right)$ contain the equations

$$
\begin{aligned}
\frac{\partial A}{\partial x^{1}}=\frac{\partial A}{\partial x^{2}} & =x^{2} \frac{\partial A}{\partial x^{1}}+\sum_{h=1}^{k} x_{h}^{2} \frac{\partial A}{\partial x_{h}^{1}}=0, \\
x^{1} \frac{\partial A}{\partial x^{2}}+\sum_{h=1}^{k} x_{h}^{1} \frac{\partial A}{\partial x_{h}^{2}} & =0
\end{aligned}
$$




$$
A=\sum_{h=1}^{k} h\left(x_{h}^{1} \frac{\partial A}{\partial x_{h}^{1}}+x_{h}^{2} \frac{\partial A}{\partial x_{h}^{2}}\right) .
$$

Suppose $A d t$ is invariant. Let $\tau:(t) \rightarrow(t, t), 0 \leqq t \leqq 1$. Then $x_{h}^{1}\left(p^{k}(\tau)\right)=x_{h}^{2}\left(p^{k}(\tau)\right)=0$ if $h>1$ and $x_{1}^{1}\left(p^{k}(\tau)\right)=x_{1}^{2}\left(p^{k}(\tau)\right)=1$.

One finds from the above equations that $A\left(p^{k}(\tau)\right)=0$. This means all the Chen constants for $\tau$ are the same as for the constant path. Since the special linear group is a subgroup of the general projective group, the same conclusion holds for that case.

(d) The existence of invariants depends on how the group acts. Let $G=$ $\{g=(a, b) \mid a \in R, b \in R\}$ and $(a, b) \cdot\left(a^{\prime}, b^{\prime}\right)=\left(a a^{\prime}, a^{\prime} b+b^{\prime}\right)$.

If $G$ acts on $E^{2}$ by $g\left(x^{1}, x^{2}\right)=\left(x^{1}, a x^{2}+b\right)$, then $\Theta$ is generated by $\partial / \partial x^{2}$ and $x^{2}\left(\partial / \partial x^{2}\right)$. As in example (c) the path $\tau:(t) \rightarrow(0, t), 0 \leqq t \leqq 1$, has $A\left(p^{k}(\tau)\right)=0$ for any invariant 1 -form $A d t$ on $J^{k}(M)$.

However, if $G$ acts by $g\left(x^{1}, x^{2}\right)=\left(a x^{1}+b, a x^{2}\right)$, then $\Theta$ is generated by $\partial / \partial x^{1}$ and $x^{1}\left(\partial / \partial x^{1}\right)+x^{2}\left(\partial / \partial x^{2}\right)$. Hence $\left(x_{1}^{1} / x^{2}\right) d t$ and $\left(x_{1}^{2} / x^{2}\right) d t$ are invariant on $J^{1}(M)$ where $x^{2} \neq 0$. The only path (not passing through $x^{2}=0$ ) on which these invariants are identically zero is the constant path.

\section{Completeness}

3.1. Algebraic theory. Chen's invariants for translation groups possess remarkable algebraic properties. Chen [4] considered paths which were piecewise "regular," i.e., had nonvanishing tangent vectors at all but a finite number of points and a finite number of jump discontinuities. Then the "product" $\tau \cdot \sigma$ of two paths $\tau$ and $\sigma$ could be defined as well as "the inverse" $\tau^{-1}$.

Such a course could be imitated here, defining "piecewise $C^{\infty}$-paths" in the obvious way. As in [4], one can prove that the Chen constants for any invariant 1 -forms under a group $G$ satisfy $\theta(\tau \cdot \sigma)=\theta(\tau) \cdot \theta(\sigma)$. However, $\theta\left(\tau \cdot \tau^{-1}\right)$ need not be 1 (cf. example (b) above). Hence $\theta\left(\tau^{-1}\right)$ need not be $\theta(\tau)^{-1}$.

3.2. Characterization. Chen's invariants in [4] characterize paths under translations: $\theta(\tau)=\theta(\sigma)$ implies $\tau$ and $\sigma$ are obtained from each other by translations and such operations as multiplication by $\gamma \cdot \gamma^{-1}$ which do not change $\theta$. Example (c) shows that this property fails in general. Next, a criterion will be given under which it does hold. (Recall that our paths are $C^{\infty}$, hence such paths as $\gamma \cdot \gamma^{-1}$ do not enter into the considerations.)

Definition 3.1. Let $A_{1} d t, \cdots, A_{r} d t$ be invariant 1 -forms under $G$ on an open set $U \subset J^{k}(M)$. Assume that for all sets $f_{1}, \cdots, f_{r}$ of $r$ real-valued $C^{\infty}$-functions defined on an open set $V \subset R$, the set

$$
\mathscr{U}=\left\{X \in \alpha^{-1}(V) \cap U \mid A_{1}(X)=f_{1}(\alpha(X)), \cdots, A_{r}(X)=f_{r}(\alpha(X))\right\}
$$

satisfies the following three conditions: 
(1) $\mathscr{U}$ is a submanifold of $\alpha^{-1}(V) \cap U$,

(2) $p^{k}(G)$ acts transitively on $\mathscr{U}$,

(3) $\Omega^{k}$, the canonical 1 -forms on $J^{k}(M)$, satisfy $\left.\operatorname{dim} \Omega^{k}\right|_{\mathcal{U}}=\operatorname{dim} \mathscr{U}-1$ at all points of $\mathscr{U}$.

Then $G$ is called complete of order $k$ with respect to $A_{1} d t, \cdots, A_{r} d t$ on $U$.

THEOREM 3.1. Let $G$ be complete of order $k$ with respect to $A_{1} d t, \cdots, A_{r} d t$ on $U$. Let $\sigma$ and $\tau$ be two $C^{\infty}$-paths on $M$ such that $p^{k}(\sigma)$ and $p^{k}(\tau)$ lie in $U$. If the Chen constants of $\sigma$ and $\tau$ with respect to $A_{1} d t, \cdots, A_{r} d t$ are identical, then $\tau=g \circ \sigma$ for some $g \in G$.

Proof. Suppose $\sigma$ and $\tau$ have domains $[a, b]$ and $[c, d]$, respectively. Define $C^{\infty}$-paths $\sigma^{\prime}$ and $\tau^{\prime}$ in $E^{r}=\left\{\left(y^{1}, \cdots, y^{r}\right)\right\}$ by

$$
\sigma^{\prime}(t)=\left(\int_{a}^{t} A_{1} \circ p_{s}^{k}(\sigma) d s, \cdots, \int_{a}^{t} A_{r} \circ p_{s}^{k}(\sigma) d s\right),
$$

$a \leqq t \leqq b ;$ and similarly for $\tau^{\prime}$.

Now, the Chen constants for $\sigma^{\prime}$ under the translation group on $E^{r}$ coincide with those for $\sigma$ with respect to $A_{1} d t, \cdots, A_{r} d t$ :

$$
\begin{aligned}
\int_{\sigma^{\prime}} d y^{i_{1}} \cdots d y^{i_{j}} & =\int_{a}^{b} A_{i_{1}} \circ p_{s}^{k}(\sigma) d s \cdots A_{i_{j}} \circ p_{s}^{k}(\sigma) d s \\
& =\int_{\sigma} A_{i_{1}} d t \cdots A_{i_{j}} d t ;
\end{aligned}
$$

similarly for $\tau^{\prime}$ and $\tau$. By hypothesis, these constants for $\sigma^{\prime}$ and $\tau^{\prime}$ are the same. Since $\sigma^{\prime}$ and $\tau^{\prime}$ are $C^{\infty}$-paths, they are irreducible, and it follows from [4, Theorem 4.1] that $\tau^{\prime}$ can be obtained from $\sigma^{\prime}$ by a translation. However, $\sigma^{\prime}(a)=(0, \cdots, 0)$ $=\tau^{\prime}(c)$. Hence $\sigma^{\prime}$ and $\tau^{\prime}$ coincide. Hence, one can change parameters so that $\sigma^{\prime}$ and $\tau^{\prime}$ are both defined on $[a, b]$ and agree completely: $\sigma^{\prime}(t)=\tau^{\prime}(t)$.

After this change, let $f_{1}(t)=A_{1} \circ p_{t}^{k}(\sigma)=A_{1} \circ p_{t}^{k}(\tau), \cdots, f_{r}(t)=A_{r} \circ p_{t}^{k}(\sigma)=A_{r} \circ p_{t}^{k}(\tau)$, for $a<t<b$. Then

$$
\mathscr{U}=\left\{X \in \alpha^{-1}((a, b)) \mid A_{1}(X)=f_{1}(\alpha(X)), \cdots, A_{r}(X)=f_{r}(\alpha(X))\right\}
$$

is a submanifold of $\alpha^{-1}((a, b))$ containing $j_{t}^{k}(\sigma)$ and $j_{t}^{k}(\tau)$ for $a<t<b$. Since $p^{k}(G)$ is transitive on $\mathscr{U}$, there exists $g \in G$ such that for any fixed $t_{0} \in(a, b), j_{t_{0}}^{k}(\tau)=j_{t_{0}}^{k}(g \circ \sigma)$.

Condition (3) of Definition 3.1 implies that the system of exterior differential forms

$$
\left\{\begin{array}{cc}
d x^{i}-x_{1}^{i} d t, & i=1, \cdots, n, \\
\cdot & \\
\cdot & \\
d x_{k-1}^{i}-x_{k}^{i} d t, & i=1, \cdots, n,
\end{array}\right.
$$


restricted to $\mathscr{U}$, is completely integrable and has unique solutions of degree 1 through each point of $\mathscr{U} . j_{t}^{k}(g \circ \sigma)$ and $j_{t}^{k}(\tau)$ are two such solutions through $t_{0}$. Therefore, $j_{t}^{k}(g \circ \sigma)=j_{t}^{k}(\tau)$ in a neighborhood of $t_{0}$. Hence $g \circ \sigma(t)=\beta\left(j_{t}^{k}(g \circ \sigma)\right.$ $=\beta\left(j_{t}^{k}(\tau)\right)=\tau(t)$ in a neighborhood of $t_{0}$. Since $t_{0}$ is arbitrary, this equality holds on $(a, b)$, and by continuity on $[a, b]$.

CoRollary. Let $\tau_{i}:\left[a_{i}, b_{i}\right] \rightarrow M, i=1,2$, be two $C^{\infty}$-paths in the plane for which $\left(x_{1}^{1} \circ p_{t}^{1}\left(\tau_{i}\right)\right)^{2}+\left(x_{1}^{2} \circ p_{t}^{1}\left(\tau_{i}\right)\right)^{2} \neq 0$ for $t \in\left[a_{t}, b_{i}\right]$. Let $s_{i}(t)$ denote arc length of $\tau_{i}$ measured from $a_{i}$ to $t, K_{i}(t)$ the curvature of $\tau_{i}$ at $t$. Then if

satisfy

$$
F_{i 1}=\frac{d s_{i}}{d t}, \quad F_{i 2}=K_{i} \frac{d s_{i}}{d t}, \quad i=1,2,
$$

$$
\int_{a_{1}}^{b_{1}} F_{1 i_{1}} d t \cdots F_{1 i_{j}} d t=\int_{a_{2}}^{b_{2}} F_{2 i_{1}} d t \cdots F_{2 i_{j}} d t
$$

for all $i_{1}, \cdots, i_{j}=1,2 ; j=1,2,3, \cdots$, then $\tau_{1}$ may be obtained from $\tau_{2}$ by a motion.

Proof. It suffices to observe that the group of motions is complete of order 2 with respect to $A_{1} d t=\left(\left(x_{1}^{1}\right)^{2}+\left(x_{1}^{2}\right)^{2}\right)^{1 / 2} d t$ and

$$
A_{2} d t=\left\{\left(x_{1}^{1} x_{2}^{2}-x_{2}^{1} x_{1}^{2}\right) /\left[\left(x_{1}^{1}\right)^{2}+\left(x_{1}^{2}\right)^{2}\right]\right\} d t
$$

on $U=\left\{X \in J^{2}(M) \mid\left(x_{1}^{1}\right)^{2}+\left(x_{1}^{2}\right)^{2} \neq 0\right\}$.

\section{BIBLIOGRAPHY}

1. K. T. Chen, Integration in free groups, Ann. of Math. 54 (1951), 147-162.

2. - Iterated integrals and exponential homomorphisms, Proc. London Math. Soc. (3) 4 (1954), 502-512.

3. - Integration of paths - geometric invariants and a generalized Baker-Housdorff formula, Ann. of Math. 65 (1957), 163-178.

4. - Integration of paths - a faithful representation of paths by noncommutative formal power series, Trans. Amer. Math. Soc. 89 (1958), 395-407.

5. C. Ehresmann, Introduction à la théorie des structures infinitesimals et des pseudo-groupes de Lie, Colloque International de Géométrie Différentielle du C.N.R.S., Paris, 1953.

6. E. Goursat, $A$ course in mathematical analysis, Vol. II, Part 2, Trans. by E. R. Hedrick and O. Dunkel, Dover, N. Y., 1917.

PrINCETON UNIVERSITY,

Princeton, New Jersey

UNIVERSITY OF WASHINGTON,

SEATtLe, WashingtoN 